\title{
Oft unnötig: Antibiotika für asthmakranke Kinder
}

\author{
Wenn sich bei Kindern mit Asthma die Atemwegssymptome ver- \\ schlechtern, werden oft unnötigerweise Antibiotika verschrieben. \\ Dies zeigt eine Studie mit nahezu 2 Millionen Kindern.
}

biotikaverbrauch, wurden jährlich 126 von 1.000 Kindern ohne Asthma mit Antibiotika behandelt. Von den asthmatischen Kindern erhielten 197 von 1.000 jedes Jahr Antibiotika. Der gleiche Trend zeigte sich in Großbritannien auf etwa doppelt so hohem Niveau: 250 von 1.000 Kinder ohne Asthma nahmen jährlich Antibiotika ein, sowie 374 von 1.000 Kindern mit Asthma.

Insgesamt lag die Wahrscheinlichkeit einer Antibiotikaverschreibung bei Kindern mit Asthma um 60\% höher.
Häufigste Ursachen für die Antibiotikaverschreibung an asthmatische Kinder waren Exazerbationen der obstruktiven Atemwegserkrankung oder eine akute Bronchitis, die in der Regel viral bedingt ist.

\section{Nicht hilfreich und womöglich sogar schädlich}

„Kinder mit schlecht kontrolliertem Asthma schlafen schlecht, bewegen sich nicht ausreichend und verschlechtern sich in den schulischen Leistungen. Deshalb muss die Asthmamedikation optimiert werden. Wir wollen aber nicht, dass Antibiotika verschrieben werden, die in dieser Situation nicht hilfreich sind und sogar schädlich sein können", so Studienautorin Dr. Esmé Baan von der Erasmus Universität in Rotterdam.

\section{- Dr. med. Dirk Einecke}

- Kongress der European Respiratory Society (ERS), Mailand, 9.-13.9.2017

\section{Globaler Trend mit Gesundheitsgefahren}

\section{E-Zigaretten: so harmlos nicht}

\section{Eine ganze Reihe von neuen Forschungsergebnissen gibt es zur E-Zigarette. Das Schädigungspotenzial scheint größer als gedacht.}

- Der Marlboro-Mann, unterwegs zu Pferd durch die Rocky Mountains, wird wohl bald eine E-Zigarette im Mundwinkel bewegen. Man schätzt, dass die E-Zigaretten-Industrie in den USA bald diejenige der konventionellen Glimmstängel überholt. Das Geschäft boomt.

Wenn E-Zigaretten als ,eher harmlos“ positioniert werden, birgt das Gefahren: Zum einen werden sie von jungen Leuten als Einstiegsdroge verwendet, zum zweiten nimmt das Heer derer zu, die doppelt rauchen, konventionell und elektronisch.

\section{E-Zigaretten versteifen die Gefäße}

Forscher um Magnus Lundbäck, Stockholm, haben Kreislaufeffekte einer E-Zi-
garetten-Exposition bei 15 gesunden Freiwilligen untersucht. E-Zigaretten führten innerhalb von nur 30 Minuten zu signifikanten Anstiegen von Blutdruck und Pulsfrequenz, die Gefäßsteifigkeit nahm um den Faktor 3 zu. E-Zigaretten ohne Nikotin verursachten diese Effekte nicht.

„Doppelraucher" leiden häufiger unter Atemwegsbeschwerden

Linnea Hedman von der Umeå Universität in Schweden berichtete über eine Studie mit 30.000 Personen. 12\% rauchten konventionelle Zigaretten und 2\% EZigaretten. 10\% der konventionellen Raucher konsumierten auch E-Zigaret- ten, im Vergleich zu 1,1\% der Ex-Raucher und 0,6\% der Nichtraucher.

Wer „doppelt“ rauchte, litt mit 56\% signifikant häufiger an respiratorischen Symptomen als reine konventionelle Raucher (46\%), reine E-Raucher (34\%) oder Nichtraucher (26\%).

\section{Was steckt drin?}

Die Arbeitsgruppe um Constantine Vardavas, Kreta, hatte bei 122 E-Zigaretten die Inhaltsstoffe analysiert. Alle Proben enthielten wenigstens eine Substanz, die als nicht gesund für die Atemwege klassifiziert ist (GHS-Klassifikation).

Vardavas präsentierte auch die Ergebnisse einer anderen Untersuchung über europaweite Trends in der Raucherentwöhnung. Nikotinersatztherapien (von $14,6 \%$ auf $12,2 \%$ ) und Raucherentwöhnungskliniken $(6,7 \%$ auf $5 \%)$ wurden unbeliebter, doch Versuche mit der E-Zigarette stiegen an (3,7\% auf 11\%).

- Dr. med. Dirk Einecke

- Kongress der European Respiratory Society (ERS), Mailand, 9.-13.9.2017Mailand 